\title{
Neuromodulation in Epilepsy and in Chronic Pain
}

\author{
Third Meeting of the Benelux Neuromodulation Society \\ Chapter of the International Neuromodulation Society
}

November 18-19, 2004, Ghent, Belgium

\author{
General Introduction to Epilepsy and Treatment \\ Modalities by Neuromodulation \\ S.A. Chkhenkeli \\ Department of Neurology, University of Chicago, \\ Chicago, Illinois, USA, and Department of Functional \\ Neurosurgery, Epilepsy Surgery Center, the \\ Saradzhishvili Institute of Clinical and Experimental \\ Neurology, Tbilisi, Georgia
}

\section{Objective}

Because the probability of resistant patients to achieve complete remission with new antiepileptic drugs appears to be less than $10 \%$, surgical interventions remain the main option to treat patients with intractable epilepsy. However, results do not remain successful enough, since only $29-65 \%$ of the patients were free of seizures after temporal lobe and/or localized neocortical resections. Intensive studies of the complex variants of mutually excitatory and inhibitory interrelations of brain structures participating in the epileptogenic process led to the proposal of the concept of epileptic systems, which develop in the brain of intractable epilepsy patients. According to this concept, not only neuronal populations with hypersynchronized neuronal activity are present, but there also may be a dynamic "epileptic system" that is represented by the functional cooperation of groups of epileptic neurons in different structures of the brain. Some of the elements making up such a system may be altered by medication or ablative and non-ablative surgical interventions. The existence of complex epileptic systems may be one of

(c) 2006 International Neuromodulation Society, 1094-7159/06/\$15.00/0 Neuromodulation, Volume 9, Number 2, 2006 143-153 the reasons of the intractability of epilepsy and the lack of success of localized resective surgery. It also emphasizes how essential the intensive study of new methods of suppression of the activity of epileptic foci is, with or without ablative surgical procedures. The concept of modulating epileptic focus activity and preventing or terminating seizure activity by focal brain stimulation is attracting considerable attention. To this point, the treatment of epilepsy by electrical neuromodulation is under the causeless deep influence of the methods of focal brain stimulation in movement disorders, without taking into consideration the substantial differences in pathophysiological mechanisms of these diseases.

\section{Materials and Methods}

Studies of the organization of epileptic systems, dynamics of epileptic activity, and inhibitory effects of therapeutic electrical stimulation of the brain were performed on more than 250 patients with implanted intracerebral electrodes. Direct inhibitory effects of neuromodulation were studied in 150 patients, and 54 of them were chronically implanted.

\section{Results}

Low-frequency $(4-8 \mathrm{~Hz})$ stimulation of the caudate nucleus and high-frequency $(50-100 \mathrm{~Hz})$ stimulation of the cerebellar dentate nucleus suppressed subclinical epileptic discharges and reduced the frequency of generalized, complex partial, and secondary generalized seizures. Stimulation of the center median (20$130 \mathrm{~Hz}$ ) shortly desynchronized the EEG and probably suppressed partial motor seizures. Direct subthreshold 
1-3 Hz stimulation of the epileptic focus may suppress rhythmic neo- and paleocortical after-discharges. Among the 54 patients, seizures were eliminated in 26 of them (48\%), improvement was achieved in 23 (43\%), and no improvement was observed in 5 patients (9\%).

\section{Conclusion}

Experimental and clinical data demonstrate the possibility of subjugation of epileptogenic processes by artificially enhanced inhibitory influences that might serve as a useful tool in the treatment of intractable and multifocal epilepsy, and might be combined with ablative epilepsy surgery. Different sites can be employed for direct brain stimulation to prevent or terminate epileptic seizures by various mechanisms of action and various final results of therapeutic stimulation. Electrical neuromodulation of epilepsy may, in the long run, stabilize the activity of epileptic systems at a lower, perhaps normal, level.

\section{Basic Neurophysiological Mechanisms in Epilepsy W. J. Wadman}

SILS-Center for NeuroScience, University of Amsterdam, Amsterdam, The Netherlands

Epilepsy is a type of neuronal hyperexcitability that involves mechanisms at both the cellular and the network level. Thereby, it is important to distinguish between epileptogenesis-being the gradual formation of epileptic tissue-and epileptic seizures, the functional reflections of the epileptic state.

Most clinical treatment modalities relate to the suppression of epileptic seizures, be it alone because extremely little is known about epileptogenesis. In the end state of epilepsy, which may take years to develop, various changes in ion-channel properties or their level of expression are contributing. These change the balance between regenerative (calcium and sodium) and stabilizing (potassium) membrane currents, often resulting in, for example, burst firing and enhanced rhythmic activity.

At the network level it is particularly the balance between (glutamatergic) excitation and (gabaergic) inhibition that plays a major role in the enhanced excitability. Mechanisms inducing plasticity exist at the level of both membrane currents and synaptic contacts that couple the cells in large networks. The role of most of these mechanisms during epileptogenesis needs to be elucidated.

In the context of neuromodulation we can speculate in which way focal deep brain stimulation may affect processes at the cellular and the network level. Both in terms of seizure generation and in counteracting the slow changes that let the brain slip from a stable functional state into massive hyper-synchronized activity.

\section{Pathophysiology of Temporal Lobe epilepsy}

E. Aronica

Department of (Neuro) Pathology, Academic Medical

Center, University of Amsterdam, The Netherlands

\section{Objective}

Temporal lobe epilepsy (TLE) is a common neurologic disorder. Resistance to pharmacologic treatment is a crucial clinical problem in patients with TLE, and epilepsy surgery represents a successful therapeutic strategy. Neuropathologic evaluation of surgical specimens from patients with TLE reveals two major pathologies: hippocampal sclerosis (HS) and focal mass lesions. HS represents the most common neuropathologic finding in surgical specimens from pharmacoresistant patients with TLE. Focal lesions, including tumors (gangliogliomas [GG] and dysembryoplastic neuroepithelial tumors [DNT]) and malformations (focal cortical dysplasia [FCD]) represent another frequent finding in TLE patients. Despite the increased understanding of the basic mechanisms of epilepsy, the cellular mechanism(s) underlying the epileptogenicity of focal lesions remain poorly understood. The access to clinically and histopathologically well-characterized neurosurgical material and the use of new molecular genetic and biological tools open new perspectives for epilepsy research.

\section{Materials and Methods}

Analysis of the gene expression and neurochemical profile of both human and experimental TLE was used to define the complex cascade of molecular processes involving the modification of the expression of a number of genes/proteins responsible for key neuronal functions.

\section{Results}

The pathogenesis of seizure activity in patients with HS comprises a variety of complex structural and cellular reorganization processes, involving both neuronal and glial cells. The epileptogenicity of focal lesions, such as GG, DNT, and FCD, seems to be related to the presence of an abnormal neuronal population, consisting of dysplastic appearing and highly differentiated cells containing, for example, neuropeptides, neurotrophins, gap-junctions, receptors for different neurotransmitters, and multidrug transporter proteins. However, neuron-glia interactions also may play a critical role in the generation of seizures. Astroglial cells, as well as astroglial-derived tumor cells, express functional receptors for a variety of neurotransmitters and may critically modulate synaptic transmission. In addition, an increasing number of observations emphasize the consistent presence of a high number of cells of the microglia/ macrophage lineage within these lesions. The presence 
of activated microglial cells in TLE specimens may contribute to epileptogenesis through production of inflammatory cytokines. The resistance to the pharmacologic treatment with a broad range of antiepileptic drugs is another characteristic of TLE. One possible general mechanism to account for this medical intractability is the inadequate drug concentration in the epileptogenic areas, as a result of the overexpression of multidrug transporter proteins.

\section{Conclusions}

The recent advances in the neuropathology of focal lesions associated with TLE will be presented and likely candidate mechanisms and molecules involved in epileptogenesis and drug resistance will be discussed.

\section{Mechanism of Action and Clinical Efficacy Ofvagus Nerve Stimulation for Epilepsy}

K. Vonck ${ }^{*, * *}$, V. De Herdt ${ }^{*}$, B. Ceulemans ${ }^{\dagger}$, H. Hauman ${ }^{\ddagger}$, B. Legros ${ }^{\S}$, L. Lagae ${ }^{\Uparrow}$, S. Dedeurwaerdere ${ }^{* * *}$,

D. Van Roost*, P. Boon,***

*Ghent University Hospital, ${ }^{\dagger}$ Epilepsy and

Rehabilitation Center for Children "Pulderbos," ${ }^{\ddagger}$ Center

for Epilepsy and Psycho-organic Disorders, Duffel,

${ }^{8}$ Erasmus Hospital, Free University Brussels, "University

Hospital Catholic University Leuven, Brussels,

**aboratory for Clinical and Experimental

Neurophysiology, Ghent University, Belgium

Vagal nerve stimulation (VNS) is a neurophysiologic treatment for patients with medically or surgically refractory epilepsy. Since the first human implant of the NCP device in 1989, more than 30,000 patients have been treated with VNS. No clear predictive factors for responders have been identified. To date, the precise mechanism of action remains to be elucidated. Better insight in the mechanism of action may identify seizure types or syndromes that respond better to VNS, may guide the search for optimal stimulation parameters, and finally improve clinical efficacy.

Animal experiments with VNS were initially performed to demonstrate efficacy and safety preceding human trials. In the search for mechanisms of action animal experiments can provide essential clues such as crucial neuranatomical structures that are involved in the seizure-suppressing effect of VNS. The nucleus of the solitary tract as well as the locus coeruleus have been identified to play such a role. However, animal experiments are often labor intensive even in the hands of experienced researchers, and the results remain only a reflection of the complicated pathophysiologic systems of the human brain. Some discrepancies between animal and human data have emerged. With the development of animal models that mimic human refractory epilepsy more reliably such as the kindling model, the status epilepeticus model, and genetic models, more valuable and usable information for human applicability may become available.
Research on the mode of action of VNS in humans is a challenge because of safety concerns, the large number of patients required, and the heterogenous nature of various small patient series. In the past 10 years some progress has been made through neurophysiologic, neuroanatomical, neurochemical, and cerebral blood flow studies in patients and animals undergoing VNS. Interesting results have been found in VNS-treated patients that underwent evoked potential measurements, cerebrospinal fluid investigation, neuropsychological testing and PET, SPECT (single photon emission computed tomography), and fMRI (functional magnetic resonance imaging) testing. Desynchronization of abnormal synchronous epileptic activity is one of the hypotheses on the mode of action that might primarily be responsible for an antiseizure effect. There is however, increasing evidence from research and clinical observation that VNS might establish a true and longterm antiepileptic effect. It has been shown that VNS influences neurotransmission in the brain and provokes long-term changes in cerebral blood flow in areas crucial for epileptogenesis such as the thalamus and medial temporal lobe structures.

Since 1995, 92 patients with refractory epilepsy have been treated with VNS at the Ghent University Hospital for at least 6 months. Mean follow-up in these patients is 39 months (7-114). More than half of the patients had a reduction of seizure frequency of at least $50 \%$. These results were compared to a population of refractory patients treated with VNS at Dartmouth Hitchcock Medical Center, Lebanon, New Haven, Connecticut, USA, showing similar efficacy and side-effects result. Within Belgium more than 150 patients have been treated with VNS in different epilepsy centers (Ghent University Hospital, Pulderbos, CEPOS, Erasmusziekenhuis, UZ Gasthuisberg Leuven). One hundred forty-four patients have a follow-up of at least 6 months. In these patients, mean monthly seizure frequency was reduced from 45 to 19 seizures per month.

Further elucidation of the mechanism of action may increase the clinical efficacy of VNS and provide inspiration for the development of new therapeutic modalities in the field of neurostimulation for refractory epilepsy. It also may support the use of VNS for other indications such as depression, pain, and obesity. VNS is an efficacious treatment for patients with refractory epilepsy who are not suitable candidates for epilepsy surgery. Prospective randomized studies comparing different stimulation paradigms and comparing VNS vs. new antiepileptic drugs are currently being conducted.

\section{Ictal Single Photon Emission Computed Tomography in Target Identification for Neuromodulation}

W. Van Paesschen

Department of Neurology, University Hospital

Gasthuisberg, Catholic University Leuven, Leuven, Belgium

The localizing value of ictal single photon emission computed tomography (SPECT) performed with cerebral blood flow (CBF) agents in patients with epilepsy 
is based on cerebral metabolic and perfusion coupling. Potential targets for neuromodulation in epilepsy are 1) the ictal onset zone; 2 ) the region of inhibition surrounding the ictal onset zone; and 3) cerebral structures at a distance from the ictal onset zone, which can modulate seizure activity.

Ictal hyperperfusion is used to localize the epileptogenic zone noninvasively, and is particularly useful in MR-negative partial epilepsy and focal cortical dysplasias. Subtraction ictal SPECT coregistered with MRI (SISCOM) improves the localization of the area of hyperperfusion. Ictal SPECT should always be interpreted in the context of a full presurgical evaluation. Early ictal SPECT injections minimize the problem of seizure propagation and of nonlocalization due to an early switch from ictal hyperperfusion to postictal hypoperfusion during brief extratemporal seizures. The degree of thresholding of SISCOM images affects the sensitivity and specificity of ictal SPECT. Postictal and interictal SPECT studies are less useful to localize the ictal onset zone.

Ictal hypoperfusion has received much less attention than ictal hyperperfusion. We will present new evidence to suggest that ictal hypoperfusion surrounding the hyperperfused ictal onset zone may represent cortical inhibition that is able to prevent seizure propagation.

Statistical parametric mapping (SPM) analysis of groups of selected ictal-interictal difference images has the potential to demonstrate the evolution of cortical, subcortical, and cerebellar perfusion changes during a particular seizure type, to study seizure gating mechanisms, and to provide new insights into the pathophysiology of seizures. Potential "distant" targets in mesial temporal lobe and frontal lobe epilepsy based on ictal SPECT studies will be discussed.

\section{Functional Magnetic Resonance Imaging During Treatment Planning for Refractory Epilepsy}

A. Tieleman*, K. Deblaere*, K. Vonck ${ }^{\dagger}$, E. Achten* *Department of Neuroradiology and ${ }^{\dagger}$ Department of Neurology, Ghent University Hospital, Belgium, GIfMI, Ghent Institute for fMRI, Belgium

Blood-oxygen-level-dependent (BOLD) functional magnetic resonance imaging (fMRI) has revolutionized the field of human brain mapping by providing the means to study neuronal activity noninvasively and with high spatial resolution (1). fMRI is beginning to find widely spread applications in clinical neuroscience. Its main clinical application is in the noninvasive mapping of sensori-motor, language, and memory functions. In epilepsy surgery this information can be used to minimize the risks of postoperative neuropsychologic deficit. Motor cortex localization with fMRI has generally been concordant with golden standard procedures such as electrocortical stimulation mapping (IoCSM) (2). It has proved particularly useful when anatomic structures are distorted by mass effects.

The intraarterial phenobarbital test (IAP) remains the golden standard for assessing language and memory, but does not provide localization information. Identification of the language-dominant hemisphere shows a very good correlation with the IAP. The highest correlation is obtained using verbal fluency tasks, which produces strong frontal activation but relatively weak temporal and parietal activation (3). fMRI language lateralization and localization has a high reliability and reproducibility, although many factors such as task choice and statistical threshold can influence reproducibility. Localization of language is far more difficult. Since most surgery is performed on the temporal lobe, a battery of language tasks is required to assess different aspects of the known language network, including the temporal language areas (4). Further, not all fMRI activation foci represent "critical" language areas, and resection of these foci may not necessarily result in clinically relevant deficits. Studies addressing this issue by comparing fMRI and IoCSM have reported encouraging results (5). Presurgical evaluation of the memory function is necessary to avoid postoperative amnesia. The sparse reports show promising results correlations between memory lateralization obtained with fMRI and IAP (6). At present, fMRI paradigms do not confidently assess the risk of postoperative amnestic syndrome. Further study is required prior to wider implementation within the clinical setting.

Functional MRI performed during a simple partial seizure (Ictal fMRI) can contribute to the localization of the ictal onset zone. Only limited cases have been reported, since this technique is restricted to selective patients with frequent ictal episodes associated with minimal movement (7). Recent technical advances have allowed simultaneous EEG/fMRI, which combines the high spatial resolution of fMRI with the high temporal resolution of fMRI (8). This new technique requires further validation and technical optimalization but has shown very promising results for localization of epileptogenic cortex.

\section{References}

1. Ogawa S, Lee TM, Kay AR, Tank DW. Brain magnetic resonance imaging with contrast dependent on blood oxygenation. Proc Natl Acad Sci 1990;87:98689872 .

2. Achten E, Jackson GD, Cameron JA, Abbott DF, Stella DL, Fabinyi GC. Presurgical evaluation of the motor hand area with functional MR imaging in patients with tumors and dysplastic lesions. Radiology 1999;210:529-538.

3. Deblaere K, Boon PA, Vandemaele P et al. MRI language dominance assessment in epilepsy patients at 1.0 T: region of interest analysis and comparison with intracarotid amytal testing. Neuroradiology 2004;46: 413-420.

4. Deblaere K, Backes WH, Hofman P et al. Developing a comprehensive presurgical functional MRI protocol for patients with intractable temporal lobe epilepsy: a pilot study. Neuroradiology 2002;44:667673.

5. FitzGerald DB, Cosgrove GR, Ronner $S$ et al. Location of language in the cortex: a comparison between functional MR imaging and electrocortical 
stimulation. AJNR Am J Neuroradiol 1997;18:15291539.

6. Deblaere K, Backes WH, Tieleman A et al. Lateralized anterior mesiotemporal lobe activation: semirandom functional MR imaging encoding paradigm in patients with temporal lobe epilepsy-initial experience. Radiology 2005;236:996-1003.

7. Krings T, Topper R, Reinges MH et al. Hemodynamic changes in simple partial epilepsy: a functional MRI study. Neurology 2000;54:524-527.

8. Gotman J, Benar CG, Dubeau F. Combining EEG and FMRI in epilepsy: methodological challenges and clinical results. J Clin Neurophysiol 2004;21:229-240.

\section{Magnetoencephalography: Principles and Perspective in Epilepsy Surgery \\ M. Levivier, P. Van Bogaert, B. Legros, X. De Tiège, D. Wikler \\ Erasmus hospital, Free University Brussels, Brussels, Belgium}

Epilepsy surgery relies on the precise identification of the brain tissue generating epilepsy activity. The integration of this information in the surgical plannings and actual procedures has improved our capacity to offer precise and reliable interventions in both lesional and nonlesional epilepsies. Over the last decade, major improvements have been obtained in this matter, mainly thanks to the development of image-guided neurosurgery. This neurosurgical concept takes advantage of the advancement in computing technology to allow online correlation of this information with the actual space during various neurosurgical procedures. In epilepsy surgery, both anatomical and functional/physiologic information should be gathered in such systems to allow optimal treatment. Indeed, planning for stereotactic (such as biopsy or electrode implantations), open neurosurgical (such as resection of epileptic foci or disconnection), and radiosurgical procedures (such as those performed with Gamma Knife) integrate such data acquired beforehand in order to target precise brain areas. Such approaches are nowadays routinely used in our center for epilepsy surgery.

Magnetoencephalography (MEG), also known as magnetic source imaging (MSI) when combined with structural magnetic resonance imaging, has developed to the point that it has now entered a wider commercial distribution and, tentatively, more routine clinical application. MEG and MSI seem to play an increasingly important role among the tools for the presurgical evaluation of epilepsy. Indeed, epilepsy studies show that MEG can accurately localize both ictal and interictal spike sources, as compared to both direct (intracranial EEG) and indirect (imaging abnormalities) data. Challenges remain with difficulties in detecting complex or deep sources, and the use of MEG data certainly deserves further validation studies, especially in the framework of its use for epilepsy surgery. In that context, epilepsy surgery, especially when invasive monitoring and work-up is needed, represents a unique opportunity to evaluate and validate the accuracy and pertinence of MEG and MSI data.

A number of diagnostic methods are used in the process of the presurgical evaluation of epilepsy, providing information on various morphological and functional aspects of the disorder, which are ultimately integrated in the final treatment decision. As mentioned earlier, this information also can be integrated in the planning and actual guidance of the neurosurgical intervention itself. In that respect, MEG and MSI, which provide a noninvasive, novel tool to localize and characterize epileptiform disturbance and to determine the significance of abnormalities seen on both structural and functional imaging, offer a new perspective in image-guided epilepsy surgery. Combined with information on the mapping of normal or eloquent brain function and with other noninvasive physiologic and anatomical information on the epileptic disorder, MEG and MSI ultimately should play a major role in a totally noninvasive evaluation, and tentatively therapeutic approach, in epilepsy surgery.

\section{Efficacy and Safety of Amygdalohippocampal Deep Brain Stimulation for Refractory Temporal Lobe Epilepsy}

D. Van Roost ${ }^{*, \neq}$, P. Boon ${ }^{\dagger, \neq}$, K. Vonck ${ }^{\dagger, \neq}, \mathrm{J}_{\text {. Caemaert }}$ Ca $^{*}$ *Department of Neurosurgery, ${ }^{\dagger}$ Department of Neurology, ${ }^{\ddagger}$ Reference Center for Refractory Epilepsy, Ghent University Hospital, Belgium

\section{Objective}

Deep brain stimulation (DBS) of different targets has recently been shown to be efficacious for refractory epilepsy. We have previously shown that chronic DBS electrodes are suitable for intracranial ictal onset localization in the medial temporal lobe. The purpose of the present study is to evaluate the efficacy and safety of long-term amygdalohippocampal (AH) DBS in patients with refractory temporal lobe epilepsy.

\section{Methods}

Eleven consecutive patients with refractory complex partial seizures (CPS), of whom nine had normal MRI (magnetic resonance imaging) findings, had bilateral AH-DBS electrodes and/or subdural grids implanted for ictal onset localization and subsequent stimulation. In patients with ictal onset in the temporal lobe, AH-DBS was initiated according to the side of ictal onset during a trial period with an external pulse generator. Patients in whom a $>50 \%$ reduction of interictal spikes and/or seizures was shown during the initial trial period had a pulse generator implanted subcutaneously in the abdomen. Prospectively, the frequency of CPS and sideeffects were carefully monitored, including bedside neuropsychologic testing. 


\section{Results}

Unilateral seizure onset in medial temporal lobe structures was found in 9 of the 11 patients; in two patients a bilateral medial temporal lobe onset was found, with unilateral preponderance in one patient and a very early contralateral involvement in another patient. In 10 of the 11 patients unilateral AH-DBS was performed; the truly bilateral patient was stimulated bilaterally. One patient did not fulfill the long-term implantation criteria and underwent a temporal lobectomy. After chronic AH-DBS in 10 patients with a mean follow-up of 22 months (range: 6-38 months), two patients had $>90 \%$ reduction in seizure frequency, five patients had a $>50 \%$ seizure reduction, and one patient is a nonresponder. In 6 of the 10 patients at least one antiepileptic drug could be tapered. None of the patients reported side-effects. In one patient the amygdalar implantation was complicated by a minor and asymptomatic amygdalar hemorrhage, which resolved within 1 week. In one patient a local infection occurred leading to generator replacement. None of the patients showed significant changes in bedside neurologic and neuropsychologic testing.

\section{Conclusions}

This open study demonstrates the efficacy of DBS in medial temporal lobe structures. More than half of the patients responded well to long-term AH-DBS. Assessment of side-effects including neuropsychologic functioning suggests that chronic AH-DBS is a safe treatment as well.

\section{Acknowledgments}

Supported by a Senior Clinical Investigator Grant, a Junior Researcher ("Aspirant") Grant, and Grants 1.5236.99 and 6.0324.02 from the Fund for Scientific Research (FWO)-Flanders; by Grant 01105399 from Ghent University Research Fund (B.O.F); and by the Clinical Epilepsy Grant Ghent University Hospital 2000-05.

\section{Anodic or Cathodic Motor Cortex Stimulation for Pain?}

J. Holsheimer, L. J. Manola

Institute for Biomedical Technology, University of Twente, Enschede, The Netherlands

\section{Objective}

In motor cortex stimulation (MCS) for central and trigeminal pain Resume leads are placed epidurally over the motor and sensory cortex. Several bipolar combinations are used to identify the cortical target corresponding to the painful body segment. The cathode giving the largest motor response is chosen for chronic stimulation. The catbode of a bipole is considered as the active electrode and the anode is assumed to be the indifferent electrode, as in spinal cord and peripheral nerve stimulation. This study aims to analyze whether this assumption is correct in MCS.

\section{Methods}

A computer model of MCS was used to calculate the response of cortical nerve fibers with different orientations to cathodic and anodic stimulation.

\section{Results}

A cortical fiber model parallel to the paddle of the epidural lead is depolarized when a catbodic pulse is applied and has the lowest possible threshold for its excitation. When the fiber model is turned toward $90^{\circ}$ (normal to the paddle) the threshold stimulus for its excitation is increased until it is hyperpolarized and gets unexcitable. When an anodic pulse is applied the fiber response is opposite: a fiber model parallel or normal to the paddle is hyperpolarized or depolarized, respectively. Opposite fiber responses are also obtained when the same fiber type is either in the wall of the central sulcus or in the precentral gyrus.

\section{Discussion}

The results are in accordance with experimental data (1-4). In anodic stimulation on the precentral gyrus pyramidal tract fibers are excited directly and at a lower threshold than in catbodic stimulation when pyramidal tract fibers are activated after a longer delay (transsynaptically). When the active electrode is above the central sulcus fiber responses are inverted.

\section{Conclusion}

When bipolar stimulation is applied the motor response is evoked under the anode. This electrode should be used as the cathode in stimulation for chronic pain relief.

\section{References}

1. Patton HD, Amassian VE. Single- and multipleunit analysis of cortical stage of pyramidal tract activation. J Neurophysiol 1954;17:345-363.

2. Phillips CG, Porter R. Unifocal and bifocal stimulation of the motor cortex. J Physiol (London) $1962 ; 162: 532-538$.

3. Hern JEC, Landgren S, Phillips CG, Porter R. Selective excitation of corticofugal neurones by surfaceanodal stimulation of the baboon's motor cortex. $J$ Physiol 1962;161:73-90. 
4. Gorman ALF. Differential patterns of activation of the pyramidal system elicited by surface anodal and cathodal cortical stimulation. J Neuropbysiol 1966;29: 547-564

\section{Somatosensory Cortex Stimulation for Deafferentation Pain}

D. De Ridder*, G. De Mulder*, S. Sunaert ${ }^{\dagger}$, A. Moller $^{\ddagger}$

*Department of Neurosurgery, University Hospital

Antwerp, Belgium, ${ }^{\dagger}$ Department of Radiology, University Hospital Leuven, Belgium, ${ }^{\ddagger}$ School of Behavioral and Brain Sciences, University of Texas at Dallas, Dallas, Texas, USA

\section{Objective}

As deafferentation pain is correlated to abnormal signal generation on different functional imaging techniques, such as magnetoencephalography (1), functional magnetic resonance imaging (fMRI) (2), and PET scan (3), this abnormal signal can be used as a target for neuromodulation in an attempt to suppress deafferentation pain.

\section{Methods}

Eight patients with intractable unilateral deafferentation pain underwent an fMRI of the somatosensory cortex to localize the area of abnormal signal generation associated with deafferentation pain. This fMRI area of abnormal signal is used as a target for neuronavigated transcranial magnetic stimulation (TMS). Five of the eight patients had a clinically beneficial effect with TMS of the contralateral primary somatosensory cortex and underwent an implantation of a cortical electrode on the primary somatosensory cortex.

\section{Results}

All patients had a statistically significant $(p<0.05)$ and three of these five patients have a clinically excellent suppression of their pain with epidural electrical stimulation; the two others improved without complete suppression of the pain. Follow-up time of the patients is 3-20 months.

\section{Discussion}

Functional imaging techniques have demonstrated a correlation between the amount of deafferentation pain and the amount of reorganization of the primary somatosensory cortex $(1,4)$ and the reversal of this reorganization on pain attenuation $(5,6)$. Deafferentation pain also corresponds to hyperactivity of the somatosensory thalamus (7) and cortex (8). We therefore attempted to modify this reorganization/hyperactivity using neuronavigation guided TMS, a technique that is capable of modulating cortical activity. If TMS is capable of suppression of deafferentation pain, the effect can be maintained by implantation of extradural electrodes overlying the area of signal abnormality on the primary somatosensory cortex.

\section{Conclusion}

These preliminary results suggest that electrical stimulation of the contralateral primary somatosensory cortex may be an option for pain control in patients presenting with intractable unilateral deafferentation pain.

\section{References}

1. Flor H, Elbert T, Knecht S et al. Phantom-limb pain as a perceptual correlate of cortical reorganization following arm amputation. Nature 1995;375:482-484.

2. Maihofner C, Schmelz M, Forster C, Neundorfer B, Handwerker HO. Neural activation during experimental allodynia: a functional magnetic resonance imaging study. Eur J Neurosci 2004;19:3211-3218.

3. Peyron R, Laurent B, Garcia-Larrea L. Functional imaging of brain responses to pain. A review and metaanalysis (2000). Neurophysiol Clin 2000;30:263-288.

4. Maihofner C, Handwerker HO, Neundorfer B, Birklein F. Patterns of cortical reorganization in complex regional pain syndrome. Neurology 2003;61:1707-1715.

5. Maihofner C, Handwerker HO, Neundorfer B, Birklein F. Cortical reorganization during recovery from complex regional pain syndrome. Neurology 2004;63:693-701.

6. Theuvenet PJ, Dunajski Z, Peters MJ, van Ree JM. Responses to median and tibial nerve stimulation in patients with chronic neuropathic pain. Brain Topogr 1999;11:305-313.

7. Rinaldi PC, Young RF, Albe-Fessard D, Chodakiewitz J. Spontaneous neuronal hyperactivity in the medial and intralaminar thalamic nuclei of patients with deafferentation pain.J Neurosurg 1991;74:415-421.

8. Llinas RR, Ribary U, Jeanmonod D, Kronberg E, Mitra PP. Thalamocortical dysrhythmia: a neurological and neuropsychiatric syndrome characterized by magnetoencephalography. Proc Natl Acad Sci 1999;96: 15222-15227.

Treatment of Chronic Pain Caused by Diabetic Peripheral Neuropathy with Spinal Cord Stimulation: Outcome in Nine Patients H. P. J. Buschman*, C. C. De $\operatorname{Vos}^{\dagger}$, H. E. van der $\mathrm{Aa}^{\ddagger}$ *Twente Institute for Neuromodulation, ${ }^{\dagger}$ Department of Clinical Physics, ${ }^{\ddagger}$ Department of Neurosurgery, Medical Spectrum Twente Hospital, Enschede, The Netherlands 


\section{Objectives}

Electrical spinal cord stimulation (SCS) has been shown effective as a therapy for different painful conditions, but the efficacy of this treatment for pain in peripheral diabetic neuropathy is not yet established. In the literature the information with this therapy in this patient group is sparse.

The primary objective of this study was to evaluate the efficacy of SCS for treatment of pain in patients with refractory peripheral diabetic neuropathy.

\section{Materials and Methods}

The study was designed as a prospective, open-label study in which each patient was his or her own control. Data were collected at baseline, during screening, at implant, and at regular intervals after initiation of therapy. Twelve diabetic patients with chronic pain who did not respond to conventional treatment were studied. The electrode was implanted in the thoracic/ lumbar epidural space. Immediate neuropathic pain relief was assessed by visual analog scale (VAS) after connecting the electrode to a percutaneous electrical stimulator.

\section{Results}

Nine subjects, two men and seven women (mean age 61 [SD 8] years, mean duration of diabetes 18 [13] years, mean duration of neuropathy 8.6 [5.5] years) had significant pain relief with the electrical stimulator and were converted to a permanent system. Statistically significant relief of neuropathic pain was achieved at 1 , 3 , and 6 months $(n=9)$. Median pain score was 77 at intake and 35 with the stimulator on at the end of study. Two patients ceased to benefit after a few months due to technical failure. In both patients the problems were resolved after connector cable revision. At the end of the study, all patients continued to experience significant pain relief and used the stimulator as an adjunctive $(n=3)$ or the sole treatment $(n=6)$ for their neuropathic pain.

\section{Conclusions}

Electrical SCS offers an effective therapy for chronic diabetic neuropathic pain. The therapy should be considered in diabetic patients with neuropathic pain who do not respond to conventional treatment.

\section{Single vs. Dual Mode Stimulation in Spinal Cord} Stimulator-What Is the Difference?

L. J. Manola, J. Holsheimer

Institute for Biomedical Technology, University of

Twente, Enschede, The Netherlands

\section{Objectives}

Stimulation with dual percutaneous leads is often used in SCS (spinal cord stimulation). Either a single generator gives pulses to both leads simultaneously (single mode), or two generators give pulses to each lead alternately (dual mode) (1). The aim was to compare theoretically the performance of these stimulation modes in terms of their ability to recruit the dorsal columns (DCs) and to evoke paresthesias.

\section{Methods}

Computer simulations (2) of dual and single mode SCS with two leads having an 8-mm center-to-center contact spacing were performed.The leads were in parallel at distances of $1,2,3$, and $4 \mathrm{~mm}$ (edge-to-edge) and symmetrical to the spinal cord midline. Stimulation with aligned guarded cathode $(+-+)$ combinations on both leads was simulated. The DC area recruited at discomfort threshold (DT, (3)) and its distribution among the left and right DC was calculated (4).

\section{Results}

As the distance between the leads was increased the recruited area declines from 2 to $0.9 \mathrm{~mm}^{2}$ in dual mode and from 3.65 to $2.8 \mathrm{~mm}^{2}$ in single mode. In single mode equal portions of both DCs are simultaneously recruited. In dual mode DC fibers are recruited mainly on the side corresponding to the active lead and changes to the opposite side as the stimulation switches to the other lead.

\section{Conclusions}

Assuming that DC recruitment is linearly related to paresthesia coverage, modeling predicts that the overall paresthesia coverage in dual mode is less than in single mode. This effect is due to a lack of superposition of the electrical fields induced by the two leads, because pulses are applied alternately to each lead. In single mode, recruitment of the median DCs is improved, most likely resulting in a more intense paresthesia sensation in lumbo-sacral dermatomes. When in dual mode stimulation there is some overlap of the DC areas recruited with each lead separately, the double pulse rate in the area of overlap is likely to result in a more intense paresthesia sensation in the corresponding dermatomes.

\section{References}

1. Devulder J, De Laat M, Rolly G. Dual channel electrostimulation in pain. Acta Neurol Belg 1998;98:195-198.

2. Holsheimer J. Computer modelling of spinal 
cord stimulation and its contribution to therapeutic efficacy (Review). Spinal Cord 1998;36:531-540.

3. He J, Barolat G, Ketcik B. Stimulation usage range for chronic pain management. Analgesia 1995; 1:75-80.

4. Manola L, Holsheimer J, Veltink PH. Technical performance of percutaneous leads for spinal cord stimulation: a modeling study. Neuromodulation 2005;8:88-99.

\section{A Role for the Dorsal Column GAT-1 Receptor in Experimental Neuropathic Pain}

M. Daemen, G. Hoogland, G. Spincemaille

Department of Neurosurgery, University Hospital

Maastricht, Maastricht, The Netherlands

\section{Objectives}

Knowledge of dorsal horn molecular changes is pivotal for the promotion of, among others, pharmacotherapeutic as well as neuromodulation-related advances in the treatment of neuropathic pain. Sciatic nerve ligation in rats (chronic constriction injury [CCI]) induces signs and symptoms that mimic human conditions of neuropathy. Central mechanisms have been implicated in the pathogenesis of neuropathic pain. Increase in neuronal excitability may be a consequence of decreased availability of spinal GABA ( $\gamma$-amino butyric acid). GABA availability can be regulated by the presence of the GABA-transporter GAT-1. This study investigates the dorsal horn expression of this transporter and its functional involvement towards pain behavior in the CCI model.

\section{Methods}

Male Lewis rats (total $n=37$ ) were subjected to CCI or to a sham procedure. A subgroup of animals were treated with the GAT-11 antagonist NO-711 (nicepotic acid). Animals were sacrificed 7 days following surgery. Behavioral testing was performed presurgery and at 7 days postsurgery. Testing included evaluation of mechanical allodynia using Von Frey filaments, thermal allodynia with a hot plate test, and observational testing of spontaneous hyperalgesia. Subsequently, protein expression assays for GAT-1 were performed (immunohistology and Western blotting).

\section{Results}

CCI induced marked increases in mechanical and thermal allodynia, as well as in spontaneous pain behavior after 7 days, while the sham procedure did not. GAT-1 was increased in superficial dorsal horn layers ipsilateral to the ligation side after 7 days. NO-711 pretreatment significantly reduced all tested pain behavior after 7 days.

\section{Conclusions}

These results suggest attenuated GABA-driven inhibition of spinal nociception due to its increased clearence from the synaptic cleft by GAT-1. Evidence for functional involvement of GAT-1 in neuropathic pain in the employed model can be derived from the observed effectiveness of NO-711 pretreatment. Further functional studies based on the concept of neurotransmitter-transporter alterations in neuropathy can lead to new and effective treatment strategies for neuropathic pain.

\section{Development of a Quality System for Treatment Ofmedically Refractory Epilepsy With Vagal Nerve Stimulation Therapy}

\section{H. P. J. Buschman*, L. J. J. C. Wagener-Schimmel ${ }^{\dagger}$,} J. $\operatorname{Ardesch}^{\dagger}$, G. Hageman ${ }^{\dagger}$, H. E. van der $\mathrm{Aa}^{\ddagger}$ *Twente Institute for Neuromodulation, ${ }^{\dagger}$ Department of Neurology, and ${ }^{\ddagger}$ Department of Neurosurgery, Medical Spectrum Twente Hospital, Enschede, The Netherlands

\section{Objectives}

To develop national standards and a continuous quality improvement system for consistency in application and in the quality of outcomes of VNS therapy in patients with medically refractory epilepsy.

\section{Materials and Methods}

A quality system is the organizatory structure, responsibilities, processes, and facilities for carrying out quality care. One of the core elements of a quality system is the systematic monitoring of the process of care via the quality circle. The quality system is being developed in a close collaboration with the participating medical specialists from several neurosurgery and epilepsy centers. Monitoring of the VNS-quality system is performed with the use of a number of selected indicators. The indicators relevant for the patient are effects on epilepsy (e.g., reduction of epileptic seizures and changes in seizure severity), effects on the functioning and well-being (e.g., EQ-5D), complications, and adverse events. Indicator areas relevant for the medical specialist are site profile and the number of performed VNS treatments.

\section{Results}

A stepwise approach was used in which the following steps were taken: selection of participating medical specialists and their centers, description of a treatment protocol, formulation of quality indicators, collection of data in a national database, and organization of feedback sessions for the participants. This information is used to adjust the process of treatment, establish 
procedures for future development, and make agreements with health care insurers.

\section{Conclusions}

Making reimbursements for VNS therapy dependent on participation in a national continuous quality improvement system creates a powerful financial incentive to continuously provide effective care in an efficient manner.

\section{Does It Matter that the Contact Impedance of Spinal Cord Stimulation Electrodes Is Variable? J. Holsheimer, L. Manola \\ Institute for Biomedical Technology, University of Twente, Enschede, The Netherlands}

\section{Objectives}

The impedance of a percutaneous SCS (spinal cord stimulation) electrode is highly variable and affects the currents injected by the various anodes (-) and cathodes $(+)$ when using a standard single-channel, constantvoltage stimulation device. The impedance is affected in particular by the position of the percutaneous lead in the epidural space and the presence of fibrous encapsulation and scar tissue (1). The aim of this study is to demonstrate the influence of variable electrode impedances on the distribution of currents among the anodes and cathodes, and thus on the characteristics of the electrical field activating spinal neural pathways.

\section{Methods}

A simple equivalent electrical network of SCS was considered. Contacts of the same polarity (cathodes and anodes) were connected in parallel and the connection between the anodes and cathodes was serial. A 1-V pulse was applied between the two ends of the circuitry. Given the impedance of each contact, the corresponding currents were calculated using Ohm's and Kirchoff's laws. An application program calculating these currents in a network of up to four contacts of the same polarity was made in Matlab.

\section{Results}

As an example we used a network consisting of two cathodes having impedances of $400 \mathrm{Ohms}$ each and two anodes with $350 \mathrm{Ohms}$ each. Three separate changes to this network were considered.

1 When the impedance of one cathode was increased twofold its current decreased by $44 \%$ while the current of the other cathode increased by $14 \%$. The anodal currents decreased by $15 \%$ each.

2 When one cathode was excluded (e.g., a broken cable), the current of the remaining cathode increased by $31 \%$, while the anodal currents decreased by $35 \%$.

3 When another cathode having a 400-Ohm impedance was added, the current of each cathode was decreased by $19 \%$, whereas the current of each anode was increased by $22 \%$.

\section{Conclusions}

These simple calculations show that in single-channel, voltage-controlled SCS an additional contact or an accidental change in just one contact impedance results in a change of the current supplied by each cathode and anode. As a consequence, the stimulation of the neural targets and thus paresthesia coverage will change.

\section{Reference}

1. Manola L, Holsheimer J, Veltink PH. Technical performance of percutaneous leads for spinal cord stimulation: a modeling study. Neuromodulation 2005;8:88-99.

The Mamillary Body and Mamillothalamic Tract Stimulation in Patients With Chronic Refractory Epilepsy: Is It Safe for Memory? Experience in Five Cases

A. Ivanoiu*, ${ }^{*}$, K. van Rijckevorsel*, B. Abu Serieh ${ }^{\dagger}$, M. de Tourtchaninoff*, G. Mary ${ }^{\S}$, C. Grandin ${ }^{\ddagger}$, T. Duprez ${ }^{\ddagger}$, C. Raftopoulos ${ }^{\dagger}$

Departments of *Neurology, ${ }^{\dagger}$ Neurosurgery, ${ }^{\ddagger}$ Radiology,

${ }^{\S}$ Neuropsychological Rehabilitation Center, and

Reference Center of Refractory Epilepsy, Saint-Luc

University Hospital, Catholic University of Louvain, Brussels, Belgium

\section{Objective}

The mamillary bodies (MB) and the mamillothalamic tracts (MTT) have been shown to be involved in seizures' propagation and expression, and a significant antiseizure effect has been obtained by stimulating or lesioning these structures $(1,2)$. Although bilateral lesions of either the hippocampus or the anterior thalamic nuclei result in devastating amnesia, a bilateral MB damage has not been shown to have a similar effect on memory $(3,4)$. Therefore, we considered the MB and MTT as potential targets for deep brain stimulation in the therapy of chronic refractory epilepsy (CRE) in a randomized, cross-over, double-blind study (5-7). The present communication focuses on safety issues, mainly on the risk for postoperative amnesia.

\section{Methods}

Five male patients with CRE ages 41-53 years were included into the study (5-7). The pre and postoperative 
cognitive evaluations were performed by a trained neuropsychologist who was blinded to stimulation status. After the implantation of the electrodes, at the beginning and at the end of each On/Off phase, as well as by the end of the study, an in-depth neuropsychologic evaluation was undertaken. Verbal and visual memories were assessed separately, and patient and relatives memory complaints were recorded. Attention, intellectual, executive, and instrumental functions were assessed as well.

\section{Results}

Two patients were within the normal range for all the cognitive domains at baseline, whereas three of them were slightly impaired. The subsequent postoperative cognitive evaluations showed some fluctuations, some of them being obviously due to seizures and postictal states. No consistent pattern of amnesia was observed by either the psychometric testing or the auto/hetero evaluation. As the study is not unblinded so far, no specific data on the stimulation effects are available.

\section{Conclusions}

No pattern of dense or persistent amnesia was observed in five consecutive cases of MB and MTT deep brain stimulation for CRE.

\section{References}

1. Mirski MA, Ferrendelli JA. Interruption of the mammillothalamic tract prevents seizures in guinea pigs. Science 1984;226:72-74.

2. Mirski MA, Ferrendelli JA. Selective metabolic activation of the mammillary bodies and their connections during ethosuximide-induced suppression of pentylenetetrazol seizures. Epilepsia 1986;27:194-203.

3. Aggleton JP, Saunders RC. The relationships between temporal lobe and diencephalic structures implicated in anterograde amnesia. Memory 1997;5:49-71.

4. Vann SD, Aggleton JP. The mammillary bodies: two memory systems in one? Nat Rev Neurosci 2004;5:35-43.

5. Raftopoulos C, van Rijckevorsel K, Abu Serieh B et al. Chronic electrical stimulation of the mammillary bodies and the mammillothalamic tracts in chronic refractory epilepsy. Neuromodulation by means of electrical brain stimulation. Neuromodulation 2004;7:148.

6. Raftopoulos C, van Rijckevorsel K, Abu Serieh B et al. Epileptic discharges in a mammillary body of a patient with refractory epilepsy. Neuromodulation 2005;8:236-240.

7. van Rijckevorsel K, Abu Serieh B, de Tourtchaninoff M, Raftopoulos C. Deep EEG recordings of the mammillary body in epilepsy patients. Epilepsia 2005; 46:781-785.

\section{Revision of Vagal Nerve Stimulation-Therapy System Electrodes: Surgical Technique and Clinical Outcome \\ H. E. van der Aa*, J. J. M. Vangeneugden ${ }^{\dagger}$, H. P. J. \\ Buschman $^{\ddagger}$ \\ *Department of Neurosurgery, Medisch Spectrum Twente Hospital, Enschede, The Netherlands, ${ }^{\dagger}$ Department of Neurosurgery, Academic Hospital St. Maarten, Duffel, Belgium, ${ }^{\ddagger}$ Twente Institute for Neuromodulation, Enschede, The Netherlands}

\section{Objectives}

Vagal nerve stimulation (VNS) for the management of intractable seizure disorders and chronic depression is increasingly being used. Concern about VNS therapy has been the possibility of revision or removal of the helical stimulating electrodes once treatment is considered ineffective or is no longer desired. Because the electrodes are wrapped around the vagus nerve, there is the potential for nerve injury during their removal. We report our experience with removal and revision of the VNS-therapy system.

\section{Materials and Methods}

We performed removal and/or revision of VNS electrodes in four patients who received long-term VNS therapy for drug-resistant epilepsy or chronic depression.

\section{Results}

All patients ( 3 men and 1 woman) were in adult age at the onset of VNS therapy. The mean duration of electrode implantation was 2.5 years (range $0.5-4.0$ years). In all patients, the old electrodes were removed completely from the nerve. One patient had the system removed because of the need for MRI (magnetic resonance imaging) of the head, and two because of lack of clinical benefit combined with severe side-effects. In one patient a new electrode was placed. No adverse events occurred intraoperatively or postoperatively.

\section{Conclusions}

Vagal nerve stimulation remains a viable option for improving seizure control in difficult to treat patients with epilepsy or improving mood in patients with chronic refractory depression. Device removal or revision as a result of insufficient clinical response and/or surgical complications such as hardware failure can be performed with success by an experienced surgeon. 